\title{
(6) OPEN ACCESS \\ Ethnic and socioeconomic variation in incidence of congenital heart defects
}

\author{
Rachel L Knowles, ${ }^{1}$ Deborah Ridout, ${ }_{1}^{1}$ Sonya Crowe, ${ }^{2}$ Catherine Bull, ${ }^{3}$ Jo Wray, ${ }^{3}$ \\ Jenifer Tregay, ${ }^{3}$ Rodney C Franklin, ${ }^{4}$ David J Barron, ${ }^{5}$ David Cunningham, ${ }^{6}$ \\ Roger C Parslow, ${ }^{7}$ Katherine L Brown ${ }^{3}$
}

\begin{abstract}
- Additional material is published online only. To view please visit the journal online (http://dx.doi.org/10.1136/ archdischild-2016-311143).

For numbered affiliations see end of article.
\end{abstract}

\section{Correspondence to} Dr Rachel L Knowles, Life Course Epidemiology and Statistics, Population Policy and Practice Programme, UCL Great Ormond St Institute of Child Health, 30 Guilford Street, London WC1N 1EH, UK; rachel.knowles@ucl.ac.uk

Received 1 May 2016 Revised 20 September 2016 Accepted 17 November 2016 Published Online First 16 December 2016

\begin{abstract}
Introduction Ethnic differences in the birth prevalence of congenital heart defects (CHDs) have been reported; however, studies of the contemporary UK population are lacking. We investigated ethnic variations in incidence of serious CHDs requiring cardiac intervention before 1 year of age.
\end{abstract}

Methods All infants who had a cardiac intervention in England and Wales between 1 January 2005 and 31 December 2010 were identified in the national congenital heart disease surgical audit and matched with paediatric intensive care admission records to create linked individual child records. Agreement in reporting of ethnic group by each audit was evaluated. For infants born 1 January 2006 to 31 December 2009, we calculated incidence rate ratios (IRRs) for CHDs by ethnicity and investigated age at intervention, antenatal diagnosis and area deprivation.

Results We identified 5350 infants (2940 (55.0\%) boys). Overall CHD incidence was significantly higher in Asian and Black ethnic groups compared with the White reference population (incidence rate ratios (IRR) $(95 \%$ Cls): Asian 1.5 (1.4 to 1.7); Black 1.4 (1.3 to 1.6)); incidence of specific CHDs varied by ethnicity. No significant differences in age at intervention or antenatal diagnosis rates were identified but affected children from non-White ethnic groups were more likely to be living in deprived areas than White children.

Conclusions Significant ethnic variations exist in the incidence of CHDs, including for specific defects with high infant mortality. It is essential that healthcare provision mitigates ethnic disparity, including through timely identification of CHDs at screening, supporting parental choice and effective interventions. Future research should explore the factors underlying ethnic variation and impact on longer-term outcomes.

\section{INTRODUCTION}

Congenital heart defects (CHDs) are the most common congenital malformation found in newborns, affecting approximately 6-8 per 1000 live births and are the most frequent cause of infant deaths from birth defects. ${ }^{12}$ Understanding the distribution of CHDs in the population is key to understanding the burden of these anomalies, including the factors influencing local case mix and severity, in order to anticipate health needs and provide effective and appropriately targeted services for the prevention and management of these conditions. The observed differences in reported birth prevalence between countries ${ }^{1}{ }^{3}$ may reflect

\section{What is already known on this topic?}

- Safe and Sustainable: Review of Children's Congenital Heart Surgery Services noted high congenital heart defect (CHD) rates and demand for CHD surgery in areas with large Asian communities.

- Higher prevalence of CHDs in babies of British Asian compared with non-Asian ethnicity has been reported in some regional studies in England.

- Studies using national paediatric audit data to investigate variation in CHD incidence across multiple ethnic groups in the ethnically-diverse contemporary UK population are lacking.

\section{What this study adds?}

- We report significant ethnic variation in the incidence of serious CHDs in English and Welsh infants, in national data set.

- We found no evidence of differential access to screening or healthcare but infants of non-white ethnicity were more likely to be living in deprived areas.

- Our findings of significant ethnic differences in CHD frequency are key to informing equitable provision of congenital heart surgery and mitigating disparity.
To cite: Knowles RL Ridout $\mathrm{D}$, Crowe $\mathrm{S}$, et al. Arch Dis Child 2017;102:496-502 causal factors, case ascertainment or the effectiveness of healthcare prevention.

The birth prevalence of specific defects varies by racial or ethnic group, ${ }^{4-7}$ and British Asian children have been reported to be at higher risk of complex CHDs than non-Asians. 89 Various factors have been proposed as influencing the association between ethnicity and health, including biology, migration, cultural and lifestyle factors, socioeconomic deprivation and inequitable access to health services. ${ }^{10} 11$ Some authors have suggested that socioeconomic disadvantage and reduced access to diagnostic services underlie ethnic differences in CHD prevalence, ${ }^{4} 1213$ and deprivation has been associated with higher risk of all congenital anomalies, including CHDs, in the UK. ${ }^{14}$ 


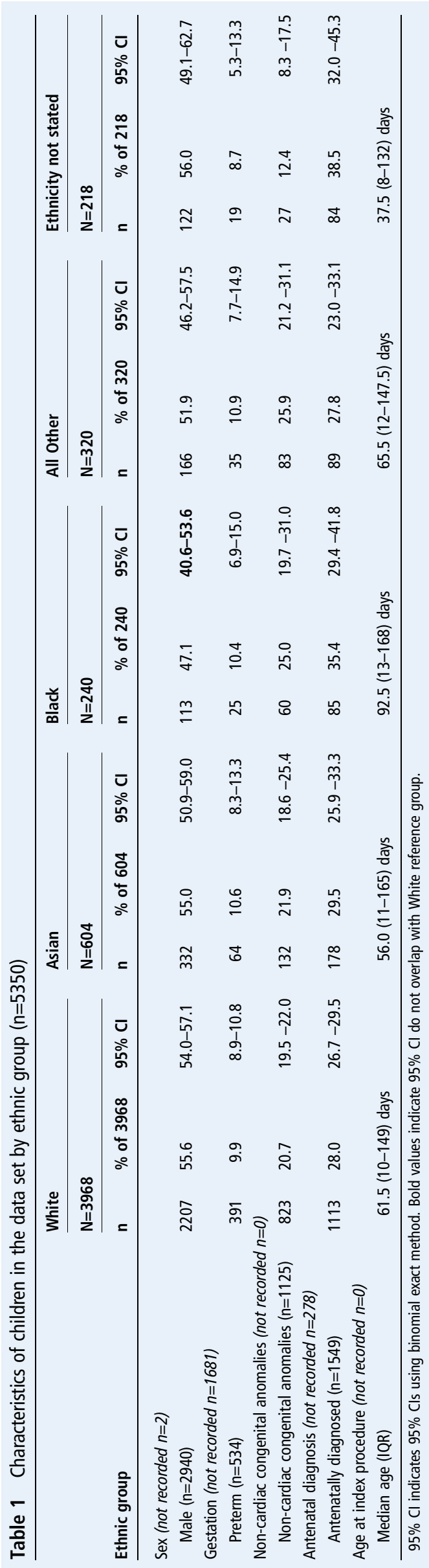

Although understanding the burden and severity of CHDs is essential to improving the quality of care and longer-term outcomes, few studies have explored ethnic variation in CHD incidence in the diverse contemporary UK population. We report here a population-based analysis, using linked routine audit data from the National Congenital Heart Disease Audit (NCHDA) and the Paediatric Intensive Care Audit Network (PICANet ${ }^{15}$ ), to investigate by ethnic group the incidence and types of CHD found in babies aged $<1$ year at the time of their first major cardiac surgery or interventional cardiac catheterisation procedure.

\section{METHODS}

Children $(n=12$ 390) aged $<1$ year who received their first major interventional cardiac procedure in England and Wales between 1 January 2005 and 31 December 2010 were identified in the $\mathrm{NCHDA}^{16}$ (formerly the National Institute for Cardiovascular Outcomes Research Congenital/Central Cardiac Audit Database). Using the National Health Service (NHS) number, we linked each child's NCHDA record to individual paediatric intensive care admissions recorded by PICANet to construct a single patient-based data set. ${ }^{17}$ We excluded 1780 infants for whom a linked PICANet record could not be identified, 2634 infants whose CHD or cardiac procedure type did not meet our eligibility criteria or who were born outside England and Wales, and 2626 infants whose index intervention was not between 1 January 2006 and 31 December 2009 (to ensure complete ascertainment for estimation of annual incidence rates (IRs)). A sensitivity analysis comparing children with and without a linked PICANet record suggested that failure to match was more likely for infants with fewer admissions, for example, those with mild CHDs (such as isolated patent ductus arteriosus (PDA)) or receiving catheter interventions.

We defined the index procedure as the first major cardiac intervention; this could be a definitive ('corrective') or palliative staging procedure, and was either an interventional cardiac catheterisation or surgical procedure. The NCHDA hierarchical classification algorithms defined a single primary diagnosis for each child. ${ }^{18}$ Babies without a diagnosis of structural CHD and premature babies (born before 37 completed weeks gestation) with an isolated PDA were excluded. An area-based deprivation score (Index of Multiple Deprivation 2010) was derived from each child's residential postcode by PICANet and subdivided into quintiles using national cut-offs. ${ }^{19}$

Ethnicity was recorded in the PICANet database using the detailed 16-category NHS ethnicity code, aggregated into six groups (see online supplementary table $\mathrm{S} 1$ ); this classification is used for the Office for National Statistics (ONS) population estimates by ethnic group. The NCHDA database used a bespoke ethnic classification: Caucasian, Asian, black, Oriental and other (including mixed ethnicity). If ethnicity differed between records for a child, the most frequent ethnic group was assigned; no child was assigned with equal frequency to more than one aggregated (six-category) ethnic group. To determine whether missing data in PICANet could be informed by the NCHDA, we evaluated concordance between the ethnic groups of children with a record of ethnicity in both audits $(n=3957)$.

Descriptive statistics are presented as numbers and percentages; 95\% CIs were estimated using the binomial exact method. IRs and incidence rate ratios (IRRs) were estimated by sex and ethnic group for all CHD and each CHD subgroup. To calculate IR, we obtained mid-year population estimates by ethnic group for children in England and Wales from the ONS. $^{20}$ 


\section{RESULTS}

Our analyses are based on 5350 children whose index intervention took place between 1 January 2006 and 31 December 2009 and for whom we had data about paediatric intensive care unit (PICU) admissions, operations and interventional catheterisations performed from birth throughout the first year of life.

\section{Ethnic classification}

No PICANet record of ethnicity was available for 1223 (22.9\%) infants, although the NCHDA recorded ethnic group for 1005 of these, of whom the majority were Caucasian $(n=717$; see online supplementary table S1). The PICANet categories White, Black, Asian, Chinese, other and mixed were paired with the NCHDA categories of Caucasian, black, Asian, Oriental and other-mixed, respectively (see online supplementary table S2). Cohen's $\kappa$ statistic for agreement was 0.81 (95\% CI 0.79 to 0.83 ) overall. The sensitivity and positive predictive value (PPV) of each NCHDA ethnic category with respect to the corresponding gold standard PICANet category ${ }^{21}$ were evaluated (see online supplementary table S3). The Caucasian, black and Asian categories in the NCHDA demonstrated good concordance $(>75 \%$ sensitivity and PPV) with the PICANet categories of White, Black and Asian, respectively, and low risk of misclassification, whereas the remaining paired categories were poorly concordant.

For further analyses, children whose PICANet ethnic group was Chinese, mixed or other were aggregated in an 'All Other' ethnic group; children whose ethnicity was missing in PICANet but recorded in the NCHDA as Caucasian, black or Asian were assigned respectively to the White, Black or Asian ethnic groups, and children coded as Oriental or 'other-mixed' in the NCHDA were assigned to 'All Other' ethnicity. We thus established a data set for further analyses in which 95.9\% $(n=5132)$ children had ethnicity data; children were grouped into White $(n=3968)$, Asian $(n=604)$, Black $(n=240)$, All Other $(n=320)$ and ethnicity not known $(n=218)$.

\section{Characteristics of infants undergoing cardiac intervention}

We identified 5350 infants with serious CHDs, of whom 2940 $(55 \%)$ were boys. Boys comprised over half of affected infants within each ethnic group except for the Black ethnic group (47\% boys). We assigned each child to one of 21 specific CHD subtypes according to the primary diagnosis. The proportion of children who had a non-cardiac congenital anomaly associated with $\mathrm{CHD}$, including syndromes, was $21.0 \%(\mathrm{n}=1125)$ and $10.0 \%(n=534)$ of infants were born preterm (before 37 completed weeks gestation). Overall $29.0 \%$ children $(n=1549)$ were diagnosed antenatally with $\mathrm{CHD}$ and the median age at index procedure was 61 (IQR 10-151) days. No significant ethnic differences in these characteristics were identified (table 1).

\section{Socioeconomic deprivation}

A higher percentage of children with CHD in non-White ethnic groups were more likely to live in the most deprived postcode areas of England and Wales. While 23.2\% of White children in the study sample lived in quintile 1 (most deprived), 51.4\% of Asian children, $53.4 \%$ of Black children and $44.2 \%$ children from All Other ethnic groups were resident in areas within the most deprived quintile (figure 1). There were also variations within aggregated ethnic groups (not shown); for example, a higher proportion of children of Asian Pakistani and Asian Bangladeshi ethnicity were living in the most deprived areas compared with those of Asian Indian ethnicity (64.3\%, 56.0\% and $35.8 \%$, respectively).

\section{Incidence of CHDs}

The incidence of children with structural CHDs who underwent a procedure within the first year of life in the UK during 20062009 was 2.0 (95\% CIs 1.9 to 2.0 ) per 1000 children aged $<1$ year ( $\mathrm{n}=5350$ infants) and did not vary significantly by year (see online supplementary table S4). The incidence was significantly higher in children of Asian and Black ethnicity (IRR 1.5 (95\% CI 1.4 to 1.7 ) and 1.4 (95\% CI 1.3 to 1.6$)$, respectively) in comparison with the reference White ethnic group (table 2; figure 2). There were no significant variations by sex within ethnic groups (figure 2).

The incidence of specific CHD subtypes varied by ethnic group (see online supplementary table S5). Compared with the White reference group, children of Asian ethnicity had
Figure 1 Distribution of cases by ethnic group and deprivation quintile $(n=4860)$. Notes: *Excludes 218 children whose ethnic group was not recorded (from Quintile [Q] 1=52, $\mathrm{Q} 2=46, \mathrm{Q} 3=37, \mathrm{Q} 4=30, \mathrm{Q}=37$, Quintile not known=16) and 272 additional children whose area deprivation score was not recorded (White=250, Asian/Black/Other=22).

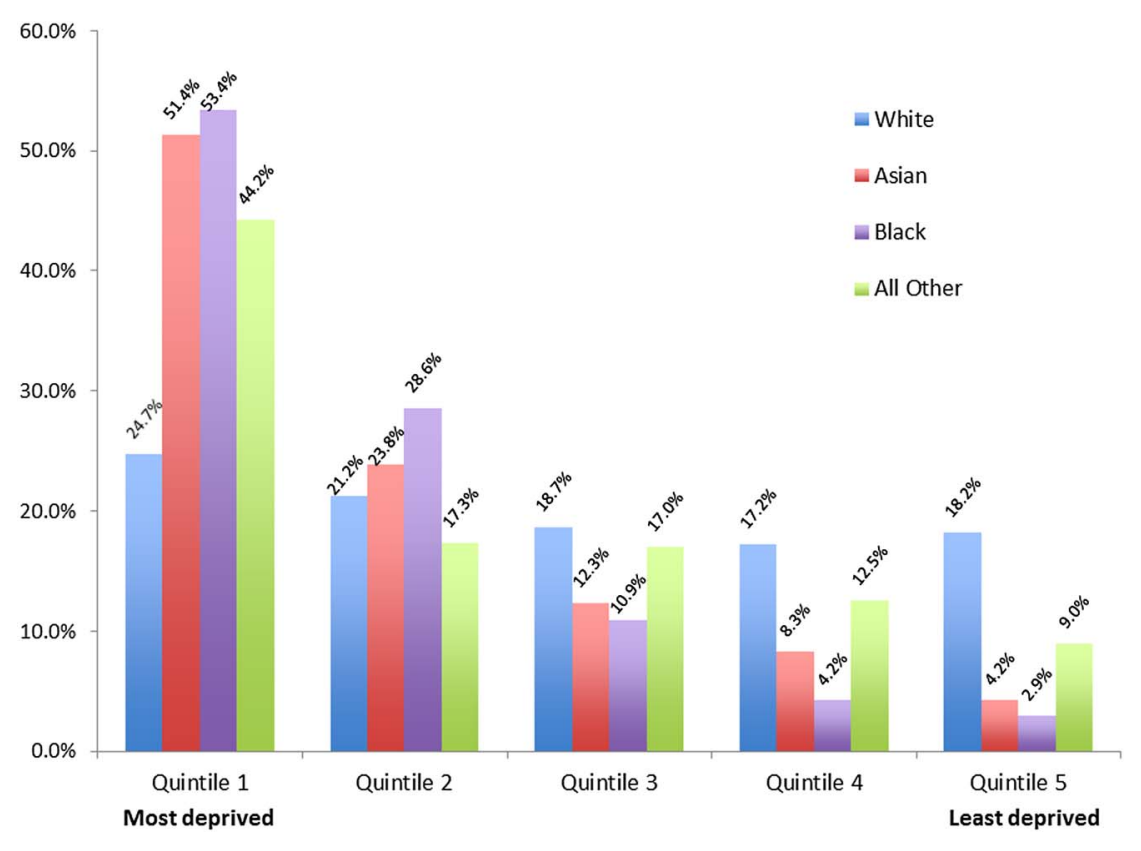

Knowles RL, et al. Arch Dis Child 2017;102:496-502. doi:10.1136/archdischild-2016-311143 
Table 2 Incidence rates and rate ratios by ethnic group (for all children undergoing index cardiac procedure, including interventional catheterisation, 2006-2009)

\begin{tabular}{|c|c|c|c|c|}
\hline & CHD cases undergoing procedure & Mid-year population aged $<1$ year* & Annual incidencet & Incidence rate ratio $(95 \% \mathrm{Cl})$ \\
\hline \multicolumn{5}{|c|}{ Ethnic group $¥(n=5132)$} \\
\hline White§ & 3968 & 2230400 & $1.8(1.7-1.8)$ & Reference \\
\hline Asianף & 604 & 220100 & $2.7(2.5-3.0)$ & $1.5(1.4-1.7)$ \\
\hline Black** & 240 & 93700 & $2.6(2.2-2.9)$ & $1.4(1.3-1.6)$ \\
\hline All Othert† & 320 & 185500 & $1.7(1.5-1.9)$ & $1.0(0.9-1.1)$ \\
\hline
\end{tabular}

${ }^{*}$ Mid-year population denominator by ethnic group for infants aged birth to 1 year in England and Wales. ${ }^{20}$

tValues are per 1000 infants with $95 \%$ Cls.

¥Rates not reported for 218 children for whom ethnicity was not stated in either source data set.

§lncludes children with ethnicity missing in PICANET but classified as Caucasian in the NCHDA.

ףIncludes children with ethnicity missing in PICANET but classified as Asian in the NCHDA.

**Includes children with ethnicity missing in PICANET but classified as black in the NCHDA.

t† Includes Chinese, mixed and other ethnicity (in PICANET) and children with ethnicity missing in PICANET but classified as Oriental or other in the NCHDA.

CHD, congenital heart defect; NCHDA, National Congenital Heart Disease Audit; PICANET, Paediatric Intensive Care Audit Network.

significantly higher IRs of functionally single ventricle, transposition of the great arteries (TGAs), pulmonary atresia (PA), tetralogy of Fallot, total anomalous pulmonary venous connection, ventricular and atrial septal defects (ASDs) and PDA; children of black ethnicity had higher rates of hypoplastic left heart (HLH), UVH, complete atrioventricular septal defects (AVSDs) and ventricular septal defects (VSD). ASDs were more common in the All Other ethnic group (table 3).

\section{DISCUSSION}

The incidence of structural CHDs requiring a cardiac interventional procedure during the first year of life is 2.0 per 1000 infants aged up to 1 year in England and Wales. Compared with the White ethnic group, the incidence of all CHDs in Asian and Black infants was around 50\% higher and for severe and complex CHD types that have high infant mortality, such as univentricular heart (UVH), HLH, TGA and PA, the incidence in Asian and Black infants was double that for White infants. This IR approximates the birth prevalence for life-threatening structural CHDs as these defects all require an interventional procedure in the first year of life; however, around 5\% of children with serious CHDs will die without undergoing any procedure. ${ }^{22}$ The proportion of infants who were diagnosed prenatally and age at first intervention do not vary significantly by ethnicity, thus there was no evidence of differential access to prenatal and postnatal diagnostic services nor clinical intervention. There were also no significant ethnic differences in the proportion of boys, infants born preterm or affected by non-cardiac congenital anomalies; however, children from non-White ethnic groups were more likely to be resident in postcode areas with higher deprivation scores.

Regional UK studies ${ }^{8}{ }^{23}$ have demonstrated higher relative rates of CHD in British Asian children similar to those evident in our national data set, including increased frequency of complex cyanotic defects and lower frequency of obstructive aortic outflow defects compared with non-Asian babies. Some authors have proposed that consanguinity contributes significantly to increased risk of congenital anomaly, ${ }^{24} 25$ particularly within the Pakistani Muslim population; however, a meta-analysis by Bittles estimated the additional anomaly risk due to consanguinity to be around $3 \% .^{26}$ Ethnic differences in CHD prevalence have been reported in north America, ${ }^{4}{ }^{6}$ but these may not be directly applicable to the UK population as the Black and Asian populations in Britain have different migratory and cultural influences to those in the USA. ${ }^{10}$ Nevertheless, international comparisons highlight the complex interplay of biological, environmental and socioeconomic factors, selective migration, cultural and lifestyle influences on the health of different ethnic groups. ${ }^{10} 1427$ They underline the importance of understanding ethnic diversity and avoiding simple categorisation into white and non-white. ${ }^{28}$

Several authors have highlighted the potential contribution of socioeconomic deprivation to increased CHD risk ${ }^{14}$ and some US
Figure 2 Incidence rate ratios of infants undergoing a cardiac intervention in England and Wales during 2006-2009 by ethnic group $(\mathrm{n}=5132)$. Notes: *Excluding 218 children with no ethnicity record; binomial exact method used to estimate confidence intervals for incidence rate ratios (IRR); reference category: White; cardiac intervention includes surgical and interventional catheter procedures.

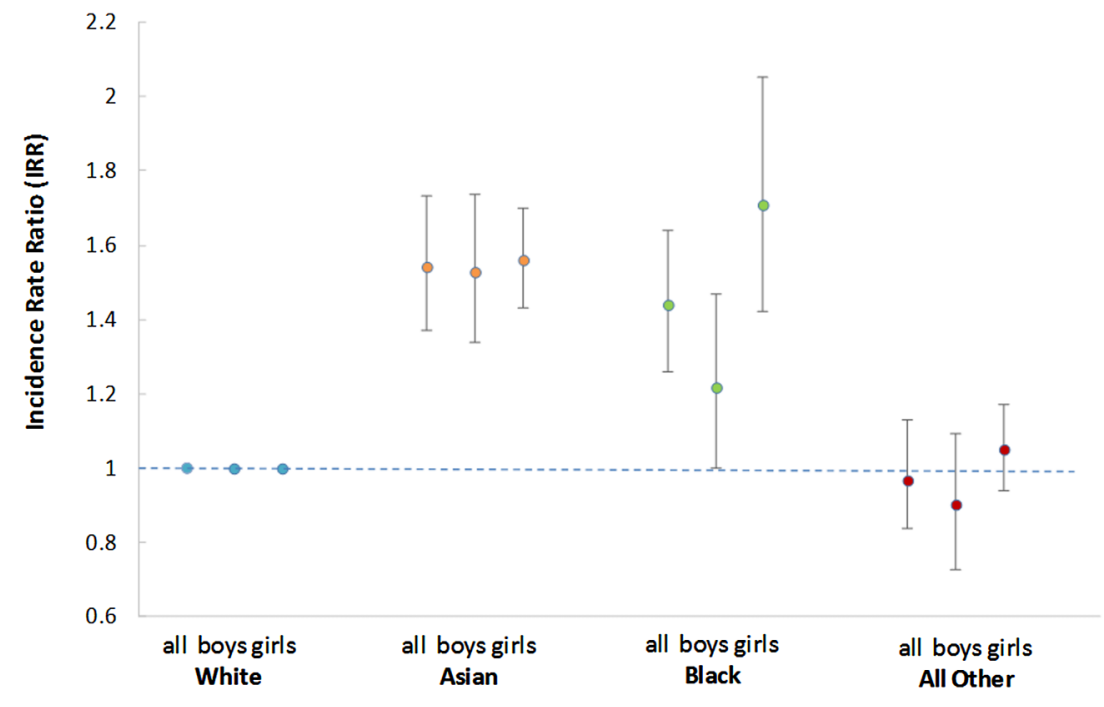




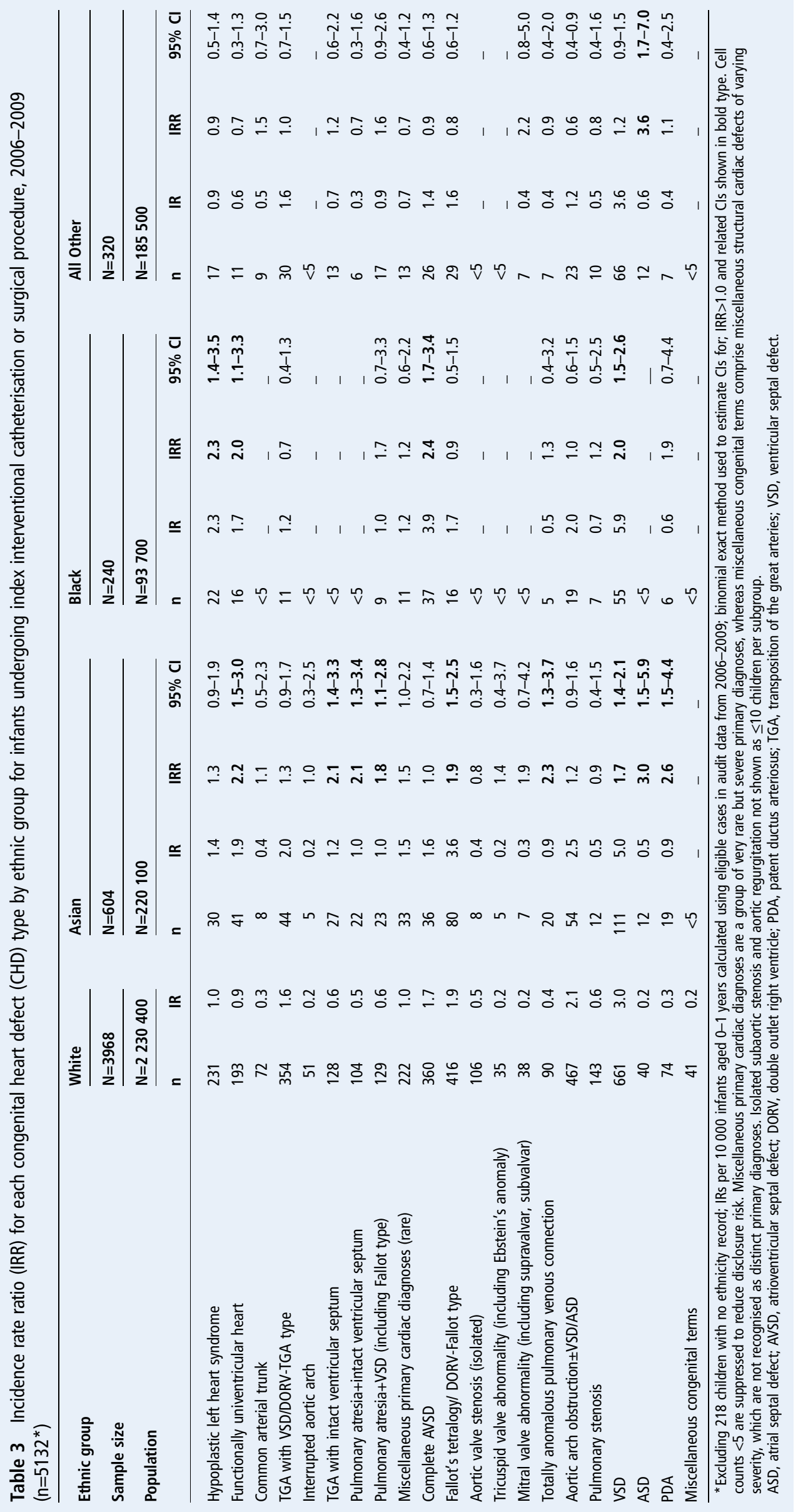


authors ${ }^{4} 2829$ have suggested that differential access to diagnostic health services within a payment-based healthcare system contributes to ethnic disparities in reported CHD prevalence. ${ }^{12} 29$ Conversely, Parslow et al have reported that mortality risk for critically ill South Asian children admitted to PICU increased as deprivation decreased. ${ }^{30}$ Nevertheless, British non-White ethnic populations are more likely to be living in more socioeconomically deprived areas; ${ }^{31}$ using our data set, we confirmed that a higher proportion of infants affected by CHDs of Black, Asian and All Other ethnicity were living in the most deprived areas of England and Wales compared with affected white infants.

Importantly prenatal diagnosis may impact on CHD prevalence at birth; in 1999, a prenatal diagnosis of serious CHD was made in $23 \%$ of all affected UK pregnancies but only $12 \%$ of infants were affected at birth; therefore, affected pregnancies ended in fetal loss or termination in around $50 \%$ of cases. ${ }^{32}$ As prenatal detection facilitates parental choice about pregnancy termination, resuscitation and rapid access to surgical intervention after delivery, it will influence birth prevalence, particularly for life-threatening CHDs detected on fetal ultrasound. ${ }^{33}$ In ethnic groups in which the most severe and life-threatening CHDs are more prevalent, pregnancy termination rates might be expected to be higher, or surgical interventions to occur earlier, than in the reference population. Where there are barriers to screening and healthcare services, babies are likely to be older at diagnosis and intervention. Hollowell et $a l^{34}$ have suggested that UK mothers of Asian Pakistani ethnicity may experience limited access to prenatal screening, and consequently lower rates of pregnancy termination; however, we found no evidence of ethnic variation in antenatal diagnosis rates. Although one study suggested that UK mothers of black ethnicity may be less likely to end a pregnancy affected by spina bifida than those of white ethnicity, ${ }^{35}$ other authors have failed to find evidence of marked ethnic differences in attitudes towards pregnancy termination for congenital anomalies 93637 and the importance of cultural, religious or ethnic influences therefore remains uncertain.

An important strength of our study was the availability of a linked national data set to support analysis of CHD frequency across a broad range of ethnic groups representative of the contemporary population of England and Wales. However, we could not extend our analysis to Northern Irish and Scottish infants due to the lack of mid-year population estimates by ethnic group for these countries. As submissions to the national audits are mandatory and externally validated, there was good case ascertainment and completion. Nevertheless, some NCHDA records could not be linked to PICANet records, and, although we found no evidence of bias, we cannot exclude the possibility that our findings may have been influenced by missing data. The ethnicity classification currently used by the NCHDA has a significant drawback as it is not comparable with many other data sets, and review of this key variable is now underway to improve monitoring of equity of access to congenital cardiac procedures in future. A further disadvantage of using routine data was that some variables were not collected, including maternal age, and other factors, such as preterm birth or comorbidities, may have been under-reported. As we lacked general population data on ethnic distribution by area deprivation within the age group of interest, we could not assess the relative contribution of deprivation and ethnicity to CHD risk; further research to understand this relationship would be merited. Encouraging improved completion of all variables is therefore crucial to improve data capture from these audits for future analyses, and consideration should be given to exploiting the potential for further linkage to fetal anomaly screening records or congenital anomaly registers, in order to inform understanding of the outcome of conceptions affected by CHDs.

Our study identified important differences in CHD prevalence in England and Wales, and confirmed that infants from Asian and Black ethnic groups are adversely affected compared with the White ethnic group. The reasons for these ethnic variations remain unclear, and, in particular, the relationship between socioeconomic deprivation and ethnicity represents an important focus for future enquiry. Further research into the natural history and outcomes after diagnosis of an affected pregnancy would provide valuable insight into the factors influencing birth prevalence of severe CHDs and postnatal survival to intervention, and would inform the equitable provision of health services to support parental choice during and after pregnancy, as well as the development of interventions to improve the outcomes of all children with CHDs.

\section{Author affiliations}

${ }^{1}$ Population Policy and Practice Programme, UCL Great Ormond St Institute of Child Health, University College London, London, UK

${ }^{2}$ Clinical Operational Research Unit, University College London, London, UK

${ }^{3}$ Cardiac Unit, Great Ormond Street Hospital NHS Foundation Trust, London, UK

${ }^{4}$ Paediatric Cardiology Department, Royal Brompton and Harefield NHS Foundation

Trust, London, UK

${ }^{5}$ Birmingham Children's Hospital NHS Foundation Trust, Birmingham, UK

${ }^{6}$ National Institute for Cardiovascular Outcomes (NICOR), London, UK

${ }^{7}$ Paediatric Intensive Care Audit Network (PICANet), Leeds, UK

Contributors RLK conceived the analysis and drafted the paper. RLK, DR and KLB designed the study, and RLK and DR performed the statistical analysis. DC and RCP undertook the linkage of NICOR and PICANET data. RLK, DR, KLB, SC, CB, JW, JT, RCF, DJB, DC and RCP contributed to the interpretation of the data. All authors revised the work critically for important intellectual content. RLK is guarantor of the paper and takes responsibility for the integrity of the work as a whole, from inception to published article.

Funding This project was funded by the National Institute for Health Research Health Services and Delivery Research programme (project no: 10/2002/29). Further information about the Infant Heart Study can be found on the following NIHR website: http:/www.nets.nihr.ac.uk/projects/hsdr/10200229. Additional support was provided by the Health Foundation, an independent charity working to continuously improve the quality of healthcare in the UK. NCHDA and PICANet are funded by the National Clinical Audit and Patient Outcomes Programme, administered by the Healthcare Quality Improvement Partnership (HQIP). PICANet is also funded by Welsh Health Specialised Services Committee; NHS Lothian/National Service Division NHS Scotland; the Royal Belfast Hospital for Sick Children; National Office of Clinical Audit Ireland (NOCA) and HCA International.

Disclaimer The views and opinions expressed herein are those of the authors and do not necessarily reflect those of the NIHR HS\&DR programme or the Department of Health.

Competing interests None declared.

Ethics approval Approval was obtained from London-Central Research Ethics Committee (reference number 12/LO/1398) and the Health Research Authority (HRA) Confidentiality Advisory Group (reference number ECC 6-02 (FT5)/2012).

Provenance and peer review Not commissioned; externally peer reviewed.

Data sharing statement The linked data set was obtained from the NCHDA and PICANET with support under Section 251 (Health and Care Act) approved by the Health Research Authority Confidentiality Advisory Group (ref: ECC 6-02(FT5)/2012). This support does not permit sharing of the study data set with third parties.

Open Access This is an Open Access article distributed in accordance with the terms of the Creative Commons Attribution (CC BY 4.0) license, which permits others to distribute, remix, adapt and build upon this work, for commercial use, provided the original work is properly cited. See: http://creativecommons.org/licenses/ by/4.0/

\section{REFERENCES}

1 van der Linde D, Konings EEM, Slager MA, et al. Birth prevalence of congenital heart disease worldwide: a systematic review and meta-analysis. J Am Coll Cardiol 2011;58:2241-7. 
2 Christianson A, Howson CP, Modell B. March of Dimes Global Report on Birth Defects: the hidden toll of dying and disabled children. White Plains, New York: March of Dimes Birth Defects Foundation, 2006.

3 Hoffman JIE. The global burden of congenital heart disease. Cardiovasc J Afr 2013;24:141-5.

4 Fixler DE, Pastor P, Sigman E, et al. Ethnicity and socioeconomic status: impact on the diagnosis of congenital heart disease. J Am Coll Cardiol 1993;21: 1722-6.

5 Canfield MA, Honein MA, Yuskiv N, et al. National estimates and race/ ethnic-specific variation of selected birth defects in the United States, 1999-2001. Birth Defects Res Part A Clin Mol Teratol 2006;76:747-56.

6 Botto LD, Correa A, Erickson JD. Racial and temporal variations in the prevalence of heart defects. Pediatrics 2001;107:E32.

7 Nembhard WN, Wang T, Loscalzo ML, et al. Variation in the prevalence of congenital heart defects by maternal race/ethnicity and infant sex. J Pediatr 2010;156:259-64.

8 Sadiq M, Stümper 0 , Wright JG, et al. Influence of ethnic origin on the pattern of congenital heart defects in the first year of life. Br Heart J 1995;73:173-6.

9 Sheridan E, Wright J, Small N, et al. Risk factors for congenital anomaly in a multiethnic birth cohort: an analysis of the Born in Bradford study. Lancet 2013;382:1350-9.

10 Davey-Smith G, Chaturvedi N, Harding S, et al. Ethnic inequalities in health: a review of UK epidemiological evidence. Crit Public Health 2000;10:375-408.

11 Sheikh A, Steiner MF, Cezard G, et al. Ethnic variations in asthma hospital admission, readmission and death: a retrospective, national cohort study of 4.62 million people in Scotland. BMC Med 2016;14:3

12 Correa-Villasenor A, McCarter R, Downing J, et al. White-black differences in cardiovascular malformations in infancy and socioeconomic factors. The Baltimore-Washington Infant Study Group. Am J Epidemiol 1991;134 393-402.

13 Egbe A, Uppu S, Stroustrup A, et al. Incidences and sociodemographics of specific congenital heart diseases in the United States of America: an evaluation of hospital discharge diagnoses. Pediatr Cardiol 2014;35:975-82.

14 Vrijheid M, Dolk H, Stone D, et al. Socioeconomic inequalities in risk of congenital anomaly. Arch Dis Child 2000;82:349-52.

15 Paediatric Intensive Care Network. Paediatric Intensive Care Audit Network: 2015 Annual Report (published November 2015). Leeds: Universities of Leeds and Leicester, 2015

16 National Institute for Cardiovascular Outcomes Research (NICOR), University College London. NICOR Congenital Heart Disease Audit website. Secondary National Institute for Cardiovascular Outcomes Research (NICOR), University College London. NICOR Congenital Heart Disease Audit website. https://nicor4.nicor.org.uk/CHD/an_ paeds.nsf/vwContent/home?Opendocument (accessed 23 Apr 2016).

17 Brown K, Wray J, Knowles RL, et al. Infant deaths in the UK community following successful cardiac surgery-building the evidence base for optimal surveillance, a mixed methods study. NIHR Journals Library. London: Queen's Printer and Controller of HMSO, 2015.

18 Brown KL, Crowe S, Pagel C, et al. Use of diagnostic information submitted to the United Kingdom Central Cardiac Audit Database: development of categorisation and allocation algorithms. Cardiol Young 2013;23:491-8.

19 English Indices of Deprivation 2010-deprivation category lookups and average scores for higher geographies. Secondary English Indices of Deprivation 2010- deprivation category lookups and average scores for higher geographies. http:/l www.apho.org.uk/resource/view.aspx?RID=110543 (accessed 10 Oct 2015).

20 Office for National Statistics. ONS mid-year Population Estimates by Ethnic Group (PEEG) mid-2009: table EE4, Population Estimates by Ethnic Group. London: Office for National Statistics, 2011.

21 Saunders $C L$, Abel GA, El Turabi A, et al. Accuracy of routinely recorded ethnic group information compared with self-reported ethnicity: evidence from the English Cancer Patient Experience survey. BMJ Open 2013;3:e002882.

22 Knowles RL, Bull C, Wren C, et al. Modelling survival and mortality risk to 15 years of age for a national cohort of children with serious congenital heart defects diagnosed in infancy. PLOS ONE 2014;9:e106806.

23 Agadoorappa P, Oddie S, Pawson N, et al. Do Pakistani babies have more congenital heart defects? Preliminary findings from our birth cohort study. Arch Dis Child 2011;96:A35.

24 Gatrad AR, Read AP, Watson GH. Consanguinity and complex cardiac anomalies with situs ambiguus. Arch Dis Child 1984;59:242-5

25 Stoltenberg C, Magnus $\mathrm{P}$, Lie RT, et al. Birth defects and parental consanguinity in Norway. Am J Epidemiol 1997;145:439-48.

26 Bittles $\mathrm{AH}$. Consanguineous marriages and congenital anomalies. Lancet 2013;382:1316-17.

27 Kucik JE, Nembhard WN, Donohue P, et al. Community socioeconomic disadvantage and the survival of infants with congenital heart defects. Am J Public Health 2014;104:e150-7.

28 Flores $\mathrm{G}$. Technical report-racial and ethnic disparities in the health and health care of children. Pediatrics 2010;125:e979-1020.

29 Gonzalez PC, Gauvreau K, Demone JA, et al. Regional racial and ethnic differences in mortality for congenital heart surgery in children May reflect unequal access to care. Pediatr Cardiol 2003:24:103-8.

30 Parslow RC, Tasker RC, Draper ES, et al. Epidemiology of critically ill children in England and Wales: incidence, mortality, deprivation and ethnicity. Arch Dis Child 2009;94:210-15.

31 Baker J, Mitchell R, Pell J. Cross-sectional study of ethnic differences in the utility of area deprivation measures to target socioeconomically deprived individuals. Soc Sci Med 2013;85:27-31.

32 Bull C. Current and potential impact of fetal diagnosis on prevalence and spectrum of serious congenital heart disease at term in the UK. British Paediatric Cardiac Association. Lancet 1999;354:1242-7.

33 Egbe A, Uppu $\mathrm{S}$, Lee $\mathrm{S}$, et al. Changing prevalence of severe congenital heart disease: a population-based study. Pediatr Cardiol 2014;35:1232-8.

34 Hollowell J, Kurinczuk JJ, Brocklehurst $P$, et al. Social and ethnic inequalities in infant mortality: a perspective from the United Kingdom. Semin Perinatol 2011;35:240-4.

35 Peake JN, Knowles RL, Shawe J, et al. Pregnancy Outcomes for Neural Tube Defect Affected Pregnancies in Different Ethnic Groups in the UK. Conference abstract B54, Society for Pediatric and Perinatal Epidemiologic Research: 27th Annual Meeting. Seattle, Washington, USA, 2014. http://sper.ssb-management.com/wp-content/ uploads/sites/2/2015/07/2014-Program.pdf (accessed 9 Sep 2016).

36 Bromiker R, Glam-Baruch M, Gofin R, et al. Association of parental consanguinity with congenital malformations among Arab newborns in Jerusalem. Clin Genet 2004;66:63-6.

37 Hewison J, Green JM, Ahmed S, et al. Attitudes to prenatal testing and termination of pregnancy for fetal abnormality: a comparison of White and Pakistani women in the UK. Prenat Diagn 2007;27:419-30. 